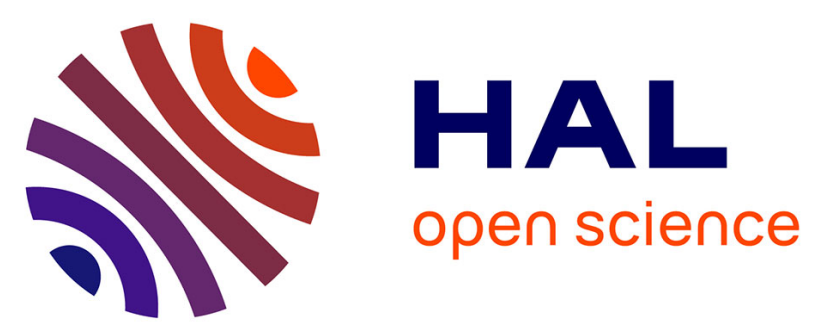

\title{
Revealing strong interactions with the reduced density gradient: a benchmark for covalent, ionic and charge-shift bonds
}

Roberto A Boto, Jean-Philip Piquemal, Julia Contreras-García

\section{- To cite this version:}

Roberto A Boto, Jean-Philip Piquemal, Julia Contreras-García. Revealing strong interactions with the reduced density gradient: a benchmark for covalent, ionic and charge-shift bonds. Theoretical Chemistry Accounts: Theory, Computation, and Modeling, 2017, 136, pp.139. hal-01636270

\author{
HAL Id: hal-01636270 \\ https://hal.science/hal-01636270
}

Submitted on 16 Nov 2017

HAL is a multi-disciplinary open access archive for the deposit and dissemination of scientific research documents, whether they are published or not. The documents may come from teaching and research institutions in France or abroad, or from public or private research centers.
L'archive ouverte pluridisciplinaire HAL, est destinée au dépôt et à la diffusion de documents scientifiques de niveau recherche, publiés ou non, émanant des établissements d'enseignement et de recherche français ou étrangers, des laboratoires publics ou privés. 


\title{
Revealing strong interactions with the reduced density gradient: a benchmark for covalent, ionic and charge-shift bonds
}

\author{
Roberto A. Boto · Jean-Philip Piquemal • Julia Contreras-García
}

Received: date / Accepted: date

\begin{abstract}
The visualization of covalent interactions has been a common practice in theoretical chemistry thanks to the Electron Localization Function (ELF). More recently, the reduced density gradient (RDG) has been introduced as a tool for revealing non-covalent interactions. Along reactions interactions change from weak to strong and vice versa. Thus, a tool enabling to analyze all of them simultaneously is fundamental for reactivity studies. This contribution is aimed at filling this gap within the NCI approach. We will highlight the ability of RDG to reveal also strong interactions and produce a benchmark that covers covalent, ionic and charge-shift bonds. The ability of the NCI descriptor to differentiate among them will be highlighted.
\end{abstract}

Keywords Visualization · Reduced density gradient · Strong interactions $\cdot$ NCI

\section{Introduction}

Density functional approximations (DFAs) have been a priceless source of bonding descriptors. The reduced density gradient (RDG or $s(\mathbf{r})$ ) was introduced in generalized gradient approximation (GGA) functionals ${ }^{1}$ as an inhomogeneity correction to the homogeneous electron gas. Therefore, it is a fundamental piece of information in GGAs.

Roberto A. Boto

CICECO - Aveiro, Institute of Materials,

University of Aveiro, 3810-193 Aveiro, Portugal

E-mail: robalboto@gmail.com

Jean-Philip Piquemal · Julia Contreras-García

Sorbonne Universités, UPMC Univ Paris 06,

UMR 7616, Laboratoire de Chimie Théorique,

F-75005, Paris, France. CNRS, UMR 7616,

Laboratoire de Chimie Théorique, F-75005, Paris, France
A non-negligible requirement for a function to lead to exchange-correlation density functionals with broad application in chemistry, is that it reveals shell structure in atoms, as well as core, bonding and long pair regions in molecular systems. In this regard RDG has been shown to reveal the exact number of atomic shells up to fifth row atoms with some exception ${ }^{2}$. Moreover, Zupan et $a l^{3}$ analyzed atomic populations within these shells and found that they yield very intuitive results. For example, Ar, $\mathrm{Li}$ and $\mathrm{Li}^{+}$core integrated populations are $10.22,2.04$ and 1.96 ; very close to the values suggested by the Aufbau principle; 10.00, 2.00 and 2.00 , respectively. In addition, they showed that the radii of the spheres containing the number of core electrons are consistent with the "ideal shell radii" computed by Schmider et al. ${ }^{4}$ Indeed, functions $|\nabla \rho| / \rho^{n}$ are able to reveal chemical bonding for a broad variety of $n$. The local-wave vector $-|\nabla \rho| / \rho$, was used by Kohout et $a l^{5}$ to distinguish atomic shells up to the sixth row. The $C_{0.6} \propto \rho^{1 / 3} s^{-1 / 2}$ index proposed by Fintzel et $a l^{6}$ leads to the populations closer to Aufbau's ones than the electron localizability indicator (ELI-D) ${ }^{7-9}$.

Moving to molecular systems, RDG has been frequently used to visualized non-covalent interactions (NCIs) within the NCI method ${ }^{10}$. Despite its original name, "NCI" for non covalent interactions, this method is not constrained to weak interactions (see Supporting Information in Ref. ${ }^{10}$ for the first examples thereto). However, applications of RDG to strong interactions are rather limited.

Bonding descriptors able to reveal different bonding situations are valuable tools for understanding chemical reactivity: chemical reactions engage the rupture and formation of chemical bonds mediated by weaker NCIs. This suggests that for a descriptor to track chemical reactions should be able to reveal from weak NCIs to 
strong ionic and covalent bonds. Recently two bonding descriptors based on the local-wave vector were put forward aiming at simultaneously describe different kinds of bonds: the localized-electrons detector ${ }^{11,12}$, the density overlap regions indicator ${ }^{13}$. Despite being the NCI method, and therefore RDG, one of the most used tools for visualizing NCIs and the evidences of its applicability to stronger interactions, a throughout study of the ability of RDG for revealing strong bonding situations is still missing.

In this work we address the problem of visualization of strong interactions by means of RDG. In Section 1 we review the topological properties of RDG and we give a chemical meaning to its critical points. Due to the absence of data, we devote Section 3 to the construction of a benchmark for RDG in strong interactions. A wide range will be covered: covalent, ionic and charge shift bonding characteristics within shall be shown. Due to their importance in organocatalysis, bonding in organometallic systems is also studied.

\section{Theory}

\subsection{The reduced density gradient}

The reduced density gradient, $s(\mathbf{r})$ is a fundamental dimensionless quantity in DFT used to describe the deviation from a homogeneous electron distribution. It is a function of the electron density, $\rho(\mathbf{r})$, and its gradient

$s(\mathbf{r})=\frac{1}{C_{s}} \frac{|\nabla \rho(\mathbf{r})|}{\rho(\mathbf{r})^{4 / 3}}$,

$C_{s}=2\left(3 \pi^{2}\right)^{1 / 3}$ and the $4 / 3$ exponent of the density ensures that $s(\mathbf{r})$ is a dimensionless quantity. Its properties as a bonding descriptor have been thoroughly highlighted within the NCI method ${ }^{10}$. Recently Boto et al proposed an interpretation of RDG as an indicator of regions where electrons exhibit bosonic behaviour ${ }^{14}$. They connected RDG with the quantity $\tau_{w}(\mathbf{r}) / \tau_{T F}(\mathbf{r})$ ${ }^{15}$, where $\tau_{T F}(\mathbf{r})=C_{F} \rho(\mathbf{r})^{5 / 3}$ is the Thomas-Fermi kinetic energy density, $C_{F}$ is the Fermi constant, and $\tau_{w}(\mathbf{r})=\frac{1}{8}|\nabla \rho(\mathbf{r})|^{2} / \rho(\mathbf{r})$ is the von Weizsäcker kinetic energy density. The latter is the kinetic energy density of a bosonic system. In one-electron systems it is identical to the positive-definite kinetic energy density $\tau(\mathbf{r})=\sum_{i} \nabla \phi_{i}^{*}(\mathbf{r}) \nabla \phi_{i}(\mathbf{r})$, where $\phi_{i}$ stands for occupied spin orbitals. In many-electron systems $\tau_{w}(\mathbf{r})$ and $\tau(\mathbf{r})$ approach each other in regions where the electron density is well described by a single orbital ${ }^{16}$. These are regions with a high probability of finding electron pairs or single electrons. Hence, minima of $\tau_{w}(\mathbf{r})$, and RDG are found in regions of marked bosonic character ${ }^{11}$, i.e. core, lone pairs and bonds.
RDG is bounded by zero from below, but has no upper boundary. It takes small values in regions where the electron density behaves similar to that of the homogeneous electron gas. It achieves its minimal value, zero, at critical points (CPs) of the electron density. Far away from nuclear positions it is driven to high values $(s(\mathbf{r}) \rightarrow \infty)$. The ability of RDG to identify relevant chemical features is reflected by its topology, that is to say, the localization and nature of its critical points. The gradient of RDG is given by the expression

$\nabla s(\mathbf{r})=\frac{\mathbf{X}\left[H(\rho(\mathbf{r}))-\frac{4}{3} \frac{|\nabla \rho(\mathbf{r})|^{2}}{\rho(\mathbf{r})} I\right]}{C_{s}|\boldsymbol{\nabla} \rho(\mathbf{r})| \rho^{4 / 3}(\mathbf{r})} \tilde{\nabla} \rho(\mathbf{r})$,

where $H(\rho(\mathbf{r}))$ is the electron density Hessian matrix, $I$ is the identity matrix of order 3 , $\mathbf{X}=\left(u_{x}, \quad u_{y}, \quad u z\right)$ contains three unit vector along directions $x, y$ and $z$ respectively and $\tilde{\nabla} \rho(\mathbf{r})$ stands for a vector with elements $\frac{\partial \rho}{\partial x}, \frac{\partial \rho}{\partial y}$ and $\frac{\partial \rho}{\partial z}$. At CPs of $\rho$ both $|\nabla \rho|$ and $\tilde{\nabla} \rho(\mathbf{r})$ elements vanish leading to a division $0 / 0$. At these points Equation 2 tends to zero along the directions $x, y, z$, and RDG displays minima. Considering this relantionship, CPs of RDG are classified differently if they are related to CPs of $\rho$ or not

1. AIM-CPs: CPs of $\rho(\mathbf{r})$, for which $\nabla \rho(\mathbf{r})=0$. These points are firmly rooted in the atoms in molecules theory (AIM) ${ }^{17}$.

2. Non-AIM-CPs: These are defined by the condition $\nabla \rho \neq 0$. Assuming that all first derivative of $\rho$ do not vanish, Non-AIM-CPs are given by the equation

$\mathbf{X}\left[H(\rho(\mathbf{r}))-\frac{4}{3} \frac{|\nabla \rho(\mathbf{r})|^{2}}{\rho(\mathbf{r})} I\right]=0$.

AIM-CPs are always minima of RDG, and they identify nuclear positions, privilege exchange channels ${ }^{18}$ and regions associated with local steric repulsion. By contrast, Non-AIM-CPs can be maxima, minima or saddle points. Under certain conditions (See Appendix) the trace of $H$, that is, the Laplacian of $\rho, \nabla^{2} \rho$, is positive and equal to $4|\nabla \rho(\mathbf{r})|^{2} / \rho(\mathbf{r})$. This condition may be written as

$\left[\frac{\nabla^{2} \rho(\mathbf{r})}{\rho(\mathbf{r})}-4\left(\frac{|\nabla \rho(\mathbf{r})|}{\rho(\mathbf{r})}\right)^{2}\right]=0$.

Two functionals of the Laplacian and the gradient of the electron density have been proposed as bonding descriptors: the one-electron potential (OEP) ${ }^{19}$ and the position uncertainty curvature (PUC $)^{20}$. Clasically allowed and forbidden regions shown negative and positive OEP values respectively. PUC has been defined as the Laplacian of $-\log (\rho(\mathbf{r}))$. This descriptor displays positive values in regions where there is a local decrease of the uncertainty electron position operator. 
Both functionals display relevant "chemical" features at their roots, such as shell structure in atoms, lone-pairs and bonding regions. Hence, it is expected that functionals of local electron density Laplacian $\left(\nabla^{2} \rho(\mathbf{r}) / \rho(\mathbf{r})\right)$ and local von Weizsäcker kinetic energy density $\left(\tau_{w}(\mathbf{r}) / \rho(\mathbf{r})\right)$ exhibit similar behavior. Left-hand side of Eq. 4 is one of these functionals and its roots are one type of Non-AIM-CP.

On the whole, critical points of RDG reveal both the gross features of the electron density distribution through AIM-CPs and the subtleties characterized by changes in the curvature of $\rho(\mathbf{r})$ as depicted by NonAIM-CPs.

\section{Computational details}

In order to asses the quality of RDG to describe strong bonds, we have selected a series of representative systems in their ground states: $\mathrm{H}_{2}, \mathrm{~N}_{2}, \mathrm{O}_{2}, \mathrm{~F}_{2}, \mathrm{CO}, \mathrm{HF}$, $\mathrm{LiH}, \mathrm{NaF}, \mathrm{NaCl}, \mathrm{KCl}, \mathrm{C}_{2} \mathrm{H}_{6}, \mathrm{C}_{2} \mathrm{H}_{4}, \mathrm{C}_{2} \mathrm{H}_{2}, t \mathrm{Bu}-\mathrm{NH}_{3}^{+}$, $t \mathrm{Bu}-\mathrm{OH}_{2}^{+},\left(\mathrm{CH}_{3}\right)_{2} \mathrm{Mg}$ and $\mathrm{CH}_{3} \mathrm{Li}$. Apart from $\mathrm{C}_{2} \mathrm{H}_{6}$, $\mathrm{C}_{2} \mathrm{H}_{4}, \mathrm{C}_{2} \mathrm{H}_{2}, \mathrm{CH}_{3} \mathrm{Li},\left(\mathrm{CH}_{3}\right)_{2} \mathrm{Mg}, t \mathrm{Bu}-\mathrm{NH}_{3}^{+}, t \mathrm{Bu}-\mathrm{OH}_{2}^{+}$ and $\mathrm{KCl}$ electron densities were calculated with the GAUSSIAN09 package ${ }^{21}$. For the rest of the system the GAMESS code $^{22}$ was used. For all the systems except $\mathrm{O}_{2}$ and $\mathrm{KCl}$ the electron density were obtained at restricted DFT level with the exchange correlation functional B3LYP and the aug-cc-pVTZ basis set. For $\mathrm{O}_{2}$, the same basis set and DFT functional was used within the unrestricted DFT formalism. Because of aug-ccpVTZ is not implemented for $\mathrm{K}$ in GAMESS, the $\mathrm{KCl}$ electron density was obtained with the TZV basis set and the restricted exchange-correlation B3LYP functional. The reduced density gradient was computed with our CHECKIN code, developed explicitly for this work and available upon request. RDG visualisation was performed with ParaView version 4.3.1 ${ }^{23}$ and VMD version $1.9 .2^{24}$.

\section{Results}

Due to the scarce literature on RDG for strong interactions and its potential use in reactivity studies, it becomes necessary to have a referent benchmark on how this function performs on different types of strong interactions. Systems such as "protocovalent" (also known as charge-shift) and organometallic have been included for their relevance in stretched situations and catalysis studies. Figures 1, 3 and 5 show the color-coded map of RDG, where the blue-green-red range goes from $s(\mathbf{r})=0$ (blue) to $s(\mathbf{r}) \geq 1$ (red). The values of RDG along the interatomic axis are also displayed.
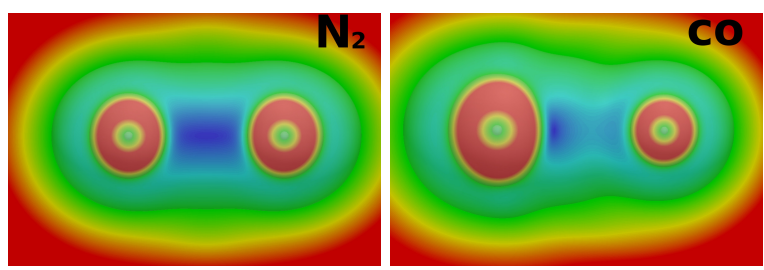

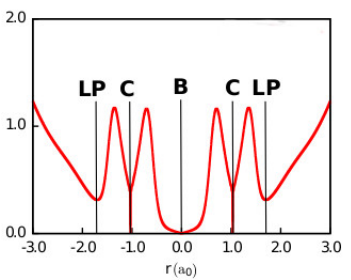

(a) $\mathrm{N}_{2}$

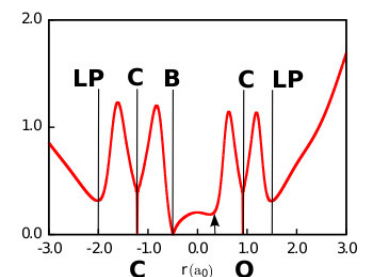

(b) $\mathrm{CO}$
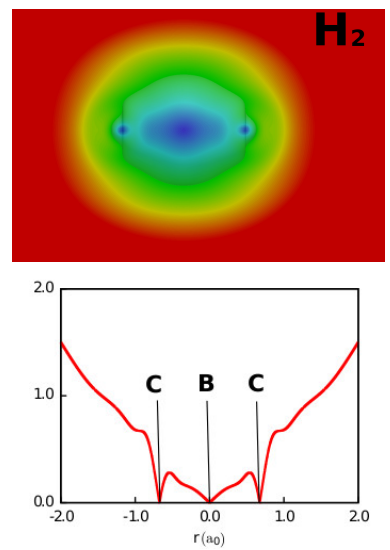

(c) $\mathrm{H}_{2}$

\section{0} 1

Fig. 1 Top panels. $s(\mathbf{r})$ contours for (a) $\mathrm{N}_{2}$, (b) CO, (c) $\mathrm{H}_{2}$, Atoms in the same order as in the notation on the topleft corner. Bottom panels. $s(\mathbf{r})$ along the internuclear axis for the same list of molecules. Labels C, B and LP in the bottom panels stand for core, bonding and lone-pair minima respectively. Arrow on $\mathrm{CO}$ plot indicates additional minima in the bonding region.

\subsection{Covalent bonds}

Figure 1(a) shows the result for $\mathrm{N}_{2}$ as a representative of purely covalent homonuclear molecule. As was previously observed, ${ }^{14} \mathrm{RDG}$ is able to recover the whole electronic pairing distribution: core, lone-pairs and interatomic bonding regions appear as minima of RDG. The bonding region in $\mathrm{N}_{2}$ corresponds to only one minimum which expands over a large region of space (both along the bonding and in the region perpendicular to it), indicating that there is a big density reconstruction upon formation of the molecule. Comparing with the $\mathrm{C}_{0.6}$ picture of $\mathrm{N}_{2}$ (Fig $4 \operatorname{Ref}^{6}$ ), there is no sign of the valence shell concentration displayed as shoulders around the BCP maximum. 
Turning to polar bonds the symmetry of the bonding region is broken. For CO (Fig. 1(b)), two minima appear. Comparing with $\mathrm{N}_{2}$ (Fig. 1(a)), we can see that the deepest minimum (AIM-CP) appears very close to the $\mathrm{C}$ atoms, whereas a second minimum (Non-AIM$\mathrm{CP}$ ) appears closer to the oxygen and corresponds to the valence shell concentration of the oxygen atom. This is the picture provided by the RDG for a very polar bond: the function is not concentrated on the center of the bond, but rather a separation appears. In the $\mathrm{CO}$ case, it is easy to see that the carbon core region is larger than that of the oxygen atom (as one would expect from the behavior along the periodic table). This also leads to a greater compressibility, yielding a compression of the carbon core along the internuclear direction. This observation is interesting from the crystallographic point of view, since it relates to recent findings on core polarization ${ }^{25}$. It is interesting to note that the $\mathrm{CO}$ case is known to provide too high charges within the QTAIM approach, which has been attributed to the displacement of the BCP towards the carbon atom, yielding a very big oxygen basin. Within the RDG approach, the extra minimum shows that a certain contribution from the oxygen to the bonding region is also important to understand the bonding in CO.

Our last example is the hydrogen molecule (Fig. 1(c)). Since there are only two electrons, $\mathrm{ELF}^{26}$ is 1 in the whole space. The analysis of RDG provides a more bonding picture. However, it is worth noting that a bonding region nearly expands from one hydrogen nucleus to the other. Since there is no Pauli repulsion from other same spin electrons, core and bonding regions are barely separated by RDG maxima in the hydrogen molecule, leading to a very diffuse electronic cloud. As previously reported ${ }^{27}$, the minima at the hydrogen positions are mathematical artifacts due to the poor performance of Gaussian functions at the atomic nuclei.

We have seen how polar bonds can be easily visualized. Let's now cast how different bond orders appear within the RDG approach. Figure 2 shows the series ethane, ethene and ethylene as the prototypical examples for bond order changes. Moving from ethane (Fig. 2(a)) to ethylene (Fig. 2(c)), the isosurface becomes closer to a cylinder as the triple bond is formed. The bond occupies bigger regions of space, as expected for the greater number of electrons it holds. It should be noted that just like the ELF analogue, the RDG picture respects the axial symmetry of the ethene molecule. However, in this case a more chemically intuitive picture is obtained (a cylinder) instead of a torus with a hole at the axis (Fig. 2(d)). It is worth noting that RDG plots along the $\mathrm{C}-\mathrm{C}$ internuclear axis only reveal signa-
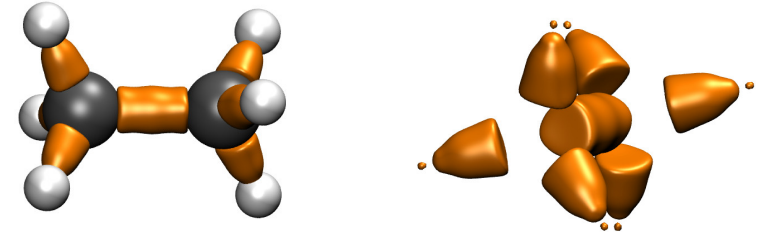

(a) $\mathrm{C}_{2} \mathrm{H}_{6}$
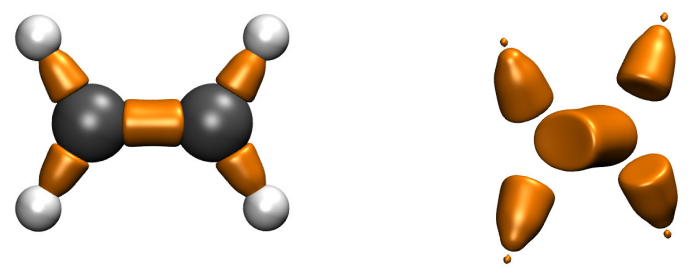

(b) $\mathrm{C}_{2} \mathrm{H}_{4}$
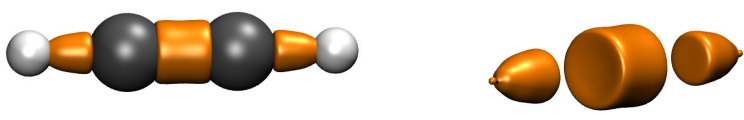

(c) $\mathrm{C}_{2} \mathrm{H}_{2}$
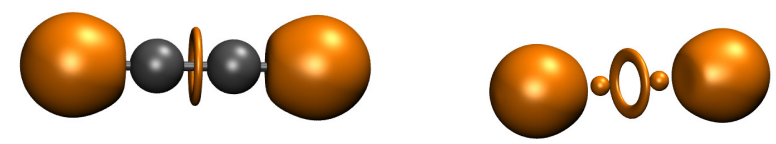

(d) $\mathrm{ELF} \mathrm{C}_{2} \mathrm{H}_{2}$

Fig. 2 (a),(b) and (c) $R D G=0.25$ isosurfaces for the ethane, ethene and ethylene series. (d) $E L F=0.88$ isosurfaces for ethylene. Molecular models are not shown at the right and the isosurfaces have been rotated in order to highlight their axial symmetry.

ture of the carbon valence shell concentration for ethane (See Fig. S2 in Supporting Information (SI)). By contrast, ethene and ethylene exhibit profiles very similar to $\mathrm{N}_{2}$, with only one minimimum at BCP. The aim of this article is to extend the framework of the visual NCI analysis to reactivity and strong bonds in general. Previous contributions ${ }^{3,6}$ have shown that these regions are accompanied by "chemical" charges. This will be the aim of a future contribution. 


\subsection{Ionic interactions}

Ionic bonding has been previously addressed within the RDG approach in Ref. ${ }^{28}$. We will add it here for completeness and to compare the results focusing on the bonding. The bonding pattern for NaF (Fig. 3(c)), represents the typical ionic case: two disconnected ions with a thin interaction region. In ionic interactions the density accumulation is very small, so just a thin surface appears around the BCP (this can be easily observed in the RDG isosurfaces contained in Fig. S1 $\mathrm{d}-\mathrm{h})$. It is interesting to note that the height of the atomic RDG curves are related to the hardness of the atoms ${ }^{28}$. This is also observed in the bonding region: the RDG curve is lower in the $\mathrm{Cl}$ compounds (see $\mathrm{NaCl}$ and $\mathrm{KCl}$ (Fig. 3(d), 3(e)), resulting surfaces that meet each other at relatively low $s$ values (ionic bond and atom). From the topological point of view, this means that the corresponding bifurcation in the bifurcation tree has a low persistance. The slope of the bonding curves seems to reflect the nature of the ions. Whereas the slopes close to the bonding basin are very steep for the cations (hard), they are smoother in the anions (soft). This leads to palatable differences in the isosurfaces, with the convex part of the surface pointing at the anion. Besides the minima at BCP, valence shell concentrations of the cation and anion are displayed close to the atomic positions.

Since H-basin are always tricky within these approaches, we have also included ionic compounds with hydrogen. To include hydrogen cation and hydride anion, figures $3(\mathrm{a})$ and $3(\mathrm{~b})$ show the bonding pattern of $\mathrm{HF}$ and $\mathrm{LiH}$ respectively. In both cases, the diffuse nature of hydrogen leads to very flat profiles and easily merged basins. The hydrogen cation and the bonding region for $\mathrm{HF}$ are completely merged with the fluorine anion valence. This image is difficult to interpret, so comparison with the covalent example $\left(\mathrm{H}_{2}\right.$, Fig. 1(c)) can help. Comparing Figures 1(c) and 3(a) we retrieve the flatness of $\mathrm{H}$, but also the deformation of the cloud towards the anion. For $\mathrm{LiH}$ the bonding region and the hydride anion are embedded in a large flat region. Following the previous reasoning, the convex surface toward the $\mathrm{H}$ atom allows to identify it as the anion of the system.

\subsection{Organometallic interactions}

Due to their relevance in organocatalysis, $\mathrm{CH}_{3} \mathrm{Li}$ and $\left(\mathrm{CH}_{3}\right)_{2} \mathrm{Mg}$ are also shown (Fig. 4(a) and 4(b)). It can be seen that these systems are a good test for previous observations. The density range at which the organometallic bonds appears correspond to intermediate strength
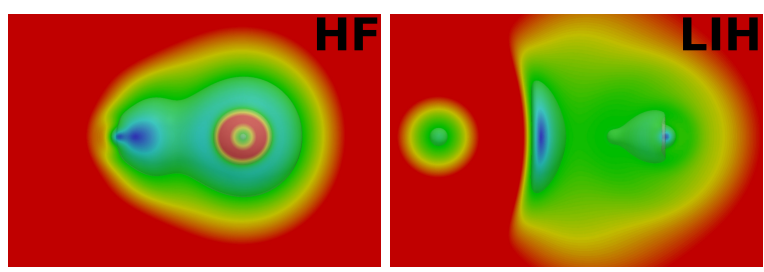

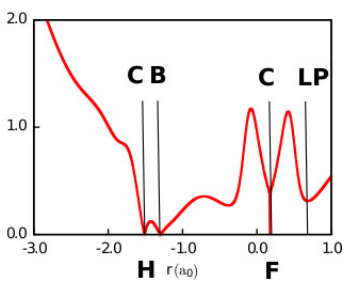

(a) $\mathrm{HF}$
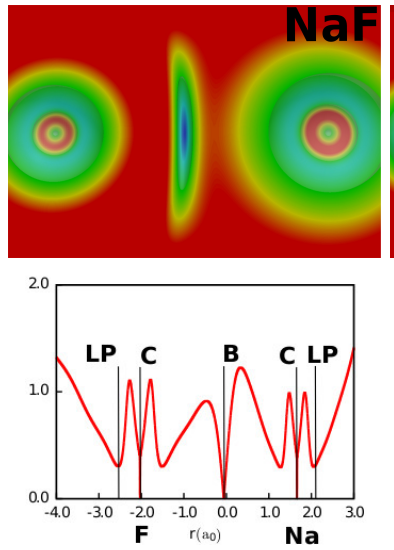

(c) $\mathrm{NaF}$

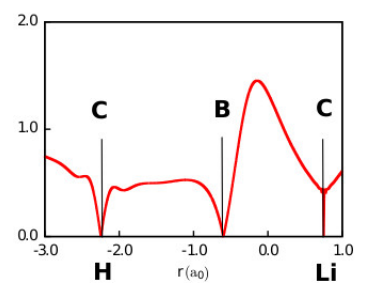

(b) $\mathrm{LiH}$
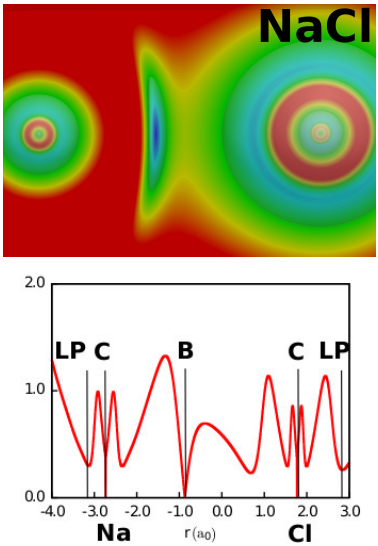

(d) $\mathrm{NaCl}$
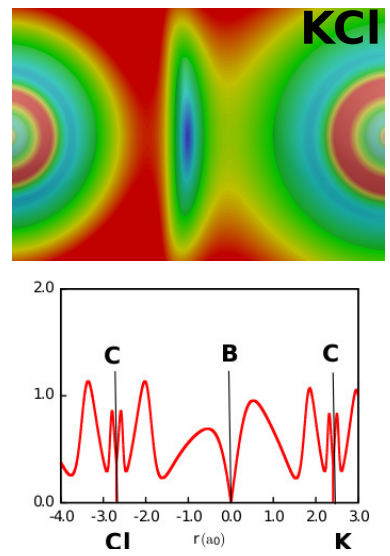

(e) $\mathrm{KCl}$

Fig. 3 Top panels. $s(\mathbf{r})$ contours for (a) HF, (b) LiH, (c) $\mathrm{NaF}$, (d) $\mathrm{NaCl}$ and (e) $\mathrm{KCl}$. Atoms in the same order as in the notation on the top-left corner. Bottom panels. $s(\mathbf{r})$ along the internuclear axis for the same list of molecules. Labels C, $\mathrm{B}$ and LP in the bottom panels stand for core, bonding and lone-pair minima respectively.

between covalent and weak interactions ( $\rho \simeq 0.05$ a.u. $)$. This is important because this means that the normal cutoff values in NCIPLOT need to be changed in order 
to visualize these interactions (See Fig. S3 for RDG vs $\rho$ plots). As far as the isosurfaces are concerned, the higher densities do not yield volumes similar to covalent cases, but rather smaller shapes similar to ionic cases. The same shape as in ionic compounds is observed, with the concave face directed toward the cation. However, the organometallic interactions appear at higher densities than ionic ones, and upon closer look, it can be seen that the orbital interaction leads to a greater elongation of the surface along the bonding direction ${ }^{12}$. All in all, the 3D signature of these bonds are intermediate, which explains their role in catalysis (directional bond, but easy to break).

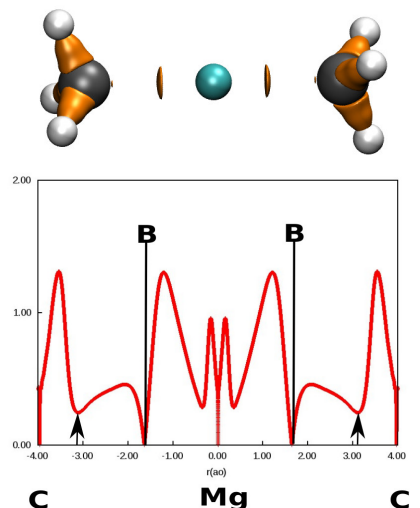

(a) $\left(\mathrm{CH}_{3}\right)_{2} \mathrm{Mg}$

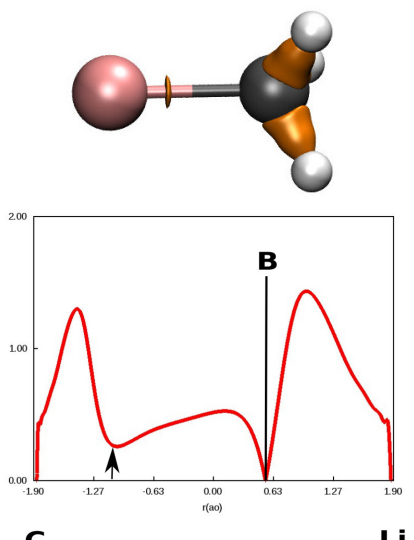

(b) $\mathrm{CH}_{3} \mathrm{Li}$

Fig. 4 (a) $\left(\mathrm{CH}_{3}\right)_{2} \mathrm{Mg}$. (Top) $0.25 \mathrm{RDG}$ isosurface and (bottom) $s(\mathbf{r})$ along the C-Mg-C axis. (b) $\mathrm{CH}_{3} \mathrm{Li}$. (Top) 0.25 RDG isosurface and (bottom) $s(\mathbf{r})$ along the $\mathrm{C}-\mathrm{Li}$ axis Label B in the $s(\mathbf{r})$ plots along internuclear axis stands for bonding minimum. Arrows indicate additional minima in the bonding region.

\subsection{Charge shift}

Charge shift bonds have been the subject of many studies in the recent literature ${ }^{29-32}$. The charge shift bond derives its stability from the resonance between covalent and ionic forms and is typically found in inverted tetrahedral carbons and even typical diatomic molecules such as $\mathrm{O}_{2}$ and $\mathrm{F}_{2}$. We have plotted these two molecules in Figures 5(a) and 5(b).
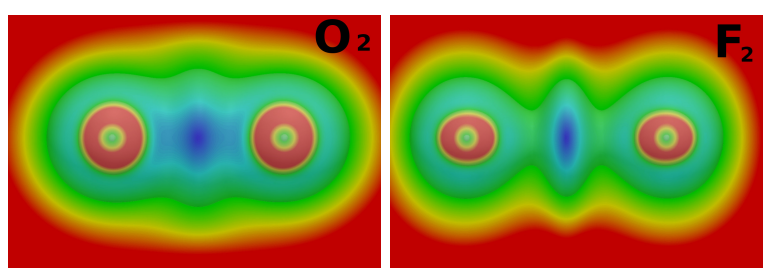

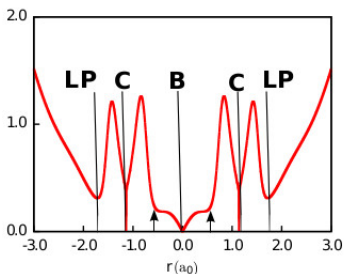

(a) $\mathrm{O}_{2}$

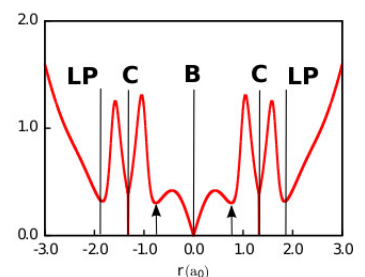

(b) $\mathrm{F}_{2}$

Fig. 5 Top panels. $s(\mathbf{r})$ contours for (a) $\mathrm{O}_{2}$, (b) $\mathrm{F}_{2}$. Atoms in the same order as in the notation on the top-left corner. Bottom panels. $s(\mathbf{r})$ along the internuclear axis for the same list of molecules. Labels C, B and LP in the bottom panels stand for core, bonding and lone-pair minima respectively. Arrows on $\mathrm{O}_{2}$ and $\mathrm{F}_{2}$ plots indicate additional minima in the bonding region.

They are characterized by a narrowing of the bonding region along the interatomic axis and an expansion along the perpendicular direction. In other words, we retrieve the minimum imposed by symmetry in the middle of the bond (coincident with the QTAIM BCP), but also extra minima closer to the involved atoms, corresponding to the valence shell concentration. Notice that this feature is missing in the nitrogen molecule (Fig 1(a) ). This picture is reminiscent of the ELF features first identified by Llusar et al in $\mathrm{F}_{2}$ as "protocovalent" bonds. ${ }^{29}$

"-Onium" systems are also representative of charge shift bonding as recently noticed by Gershoni-Poranne and Chen ${ }^{33}$. We have plotted RDG for two representative "- onium" systems: $t \mathrm{Bu}-\mathrm{NH}_{3}^{+}$and $t \mathrm{Bu}-\mathrm{OH}_{2}^{+}$. C-N and $\mathrm{C}-\mathrm{O}$ bond in these systems have been reported to exhibit high charge-shift character. As shown in Figure 6, RDG isosurface is able to differentiate between charge shift bonds $\mathrm{C}-\mathrm{N}$ and $\mathrm{C}-\mathrm{O}$, and covalent $\mathrm{C}-\mathrm{C}$ bonds. The former are characterized by a narrow isosur- 
face along the interatomic axis and a stretch along the perpendicular directions, similar to that observed for $\mathrm{F}_{2}$ and $\mathrm{O}_{2}$. The latter are extended along the interatomic region. This difference arises from the emergence of the valence shell concentration close to the central carbon atom.

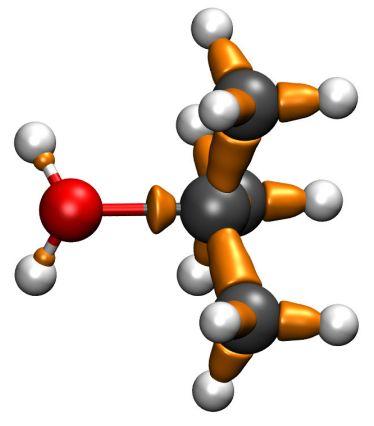

(a) $t \mathrm{Bu}-\mathrm{OH}_{2}^{+}$

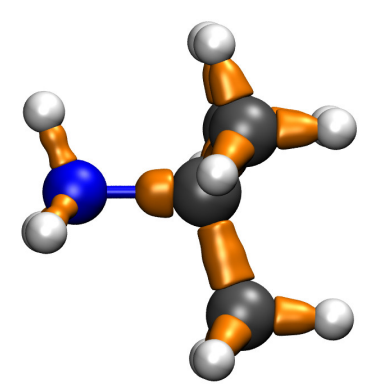

(b) $t \mathrm{Bu}-\mathrm{NH}_{3}^{+}$
Fig. $6 \mathrm{RDG}=0.25$ isosurfaces for (a) $t \mathrm{Bu}-\mathrm{OH}_{2}^{+}$and $(\mathrm{b}) t \mathrm{Bu}-$ $\mathrm{NH}_{3}^{+}$.

\subsection{Connection with other electron density inhomogeneity measures}

Recently Kohout and Wagner ${ }^{34}$ put forward a inhomogeneity measure $I_{p}$ defined as the distance in the space $L_{p}\left(\mu_{i}\right)$ from the average charge density within a region $\mu_{i}$

$I_{p}(i)=\sqrt[p]{\int_{\omega_{i}}\left|\rho(\mathbf{r})-\bar{\rho}_{i}(\mathbf{r})\right|^{p}} d V$,

where $\bar{\rho}_{i}$ is the average density in the region $\mu_{i}$. After a series of approximation and sampling the electron density on regions of fixed amount of inhomogeneity, Fintzel and Kohout $^{6}$ derived the $C_{p}$ functionals (see the original reference for a complete derivation):

$C_{p}(\mathbf{r}) \approx \rho(\mathbf{r})\left[\frac{2(p+1)^{1 / p}}{|\nabla \rho(\mathbf{r})|}\right]^{3 p /(p+3)}$.

The parameter $p$ was fixed to 0.6 in order to reproduce the topology of ELI-D in molecules. Thus $C_{0.6}$ is given by the formula:

$C_{0.6}(\mathbf{r})=\frac{8}{5^{5 / 6}} \sqrt{\rho(\mathbf{r})}\left[\frac{\rho(\mathbf{r})}{|\nabla \rho(\mathbf{r})|}\right]^{1 / 2}$.

Because of the electron density gradient is in the denominator of $C_{0.6}$, maxima of this functional emerge at CPs of $\rho$. In addition they found that valence shell electron density concentration and lone-pairs are displayed as maxima of $C_{0.6}$. This difference between maxima at electron density CPs and maxima corresponding with valence-shell concentrations is similar to our separation between AIM and Non-AIM CPs. In molecular systems the number of maxima strongly depends on the separability between the valence and the bonding region. In non-polar bonds BCP maximum and valence maxima overlap leading to a wide maximum at BCP position. Polar bonds are characterized by two maxima: one at $\mathrm{BCP}$ and other corresponding to the valence of the most electronegative peer. Non-bonding situations such as rare gases dimers, are displayed as three maxima: two valence maxima and one at BCP. Given the similarity between $C_{0.6}$ and RDG:

$C_{0.6}(\mathbf{r})=\frac{8}{5^{5 / 6} C_{s}^{1 / 2}}\left(\frac{\rho(\mathbf{r})^{1 / 3}}{s(\mathbf{r})^{1 / 2}}\right)$,

a match between $C_{0.6}$ maxima and RDG minima is expected. However, some differences might appear in non-AIM critical points, as we have seen for $\mathrm{N}_{2}$.

\section{Conclusions}

Tools to characterize covalent and non-covalent interactions on equal footing are very demanded in quantum chemistry due to their relevance for reactivity analyses. DFAs and chemical bonding theories have run parallel in this regard: bonding descriptors with such features are frequently incorporated in DFA functionals ${ }^{35}$. One of these descriptors is RDG. Despite the fact that it exhibits shell structure in atoms and reveals noncovalent interactions, a benchmark of its capabilities for visualizing strong interactions was missing. In this work we focus on the fact that RDG is a suitable scalar field for analyzing NCIs as well as strong intramolecular interactions i.e. covalent, charge-shift and ionic and organometallic bonding.

All in all, NCI not only reveals non-covalent interactions as already pointed out in previous contributions ${ }^{10,28}$. The type of bonding can be casted very easily from the mere analysis of the RDG at large densities. Moreover, it enables to identify the participation of each atom in the bonding (hard cations, soft anions, etc). Thus, the ability to reveal both covalent and noncovalent features makes of RDG an ideal tool to follow reactivity as has been seen $\operatorname{Refs}^{36-39}$.

\section{Acknowledgements}

We are grateful to the reviewers of our paper for their careful reading and helping to improve this manuscript. This work was supported partially by the framework of 
CALSIMLAB under the public grant ANR-11-LABX0037-01 overseen by the French National Research Agency (ANR) as part of the "Investissements d'Avenir" program (reference: ANR-11-IDEX-0004-02).

\section{Appendix: Critical points of $s$}

We now develop the gradient of $s$ to shed some chemical insight on the critical points of $s$. CPs of $s$ are given by the following equation:

$\nabla s=\frac{1}{C_{s}} \sum_{i, j}\left[\frac{H_{i j}}{\rho^{4 / 3}|\nabla \rho|}-\frac{4}{3} \frac{|\nabla \rho|}{\rho^{7 / 3}}\right]\left(\frac{\partial \rho}{\partial x_{j}}\right) \mathbf{u}_{\mathbf{j}}=0$,

where $x_{i}$ stands for $\{x, y, z\}, \mathbf{u}_{\mathbf{i}}$ for three unit vectors along directions $x, y$ and $z$, and $H_{i j}$ for the electron density Hessian matrix elements $\frac{\partial^{2} \rho}{\partial x_{i} x_{j}}$. Since at CPs of $s \nabla s$ must be zero along each direction $x, y$ and $z$, the following three equalities must be satisfied:

$\frac{1}{\rho^{4 / 3}|\nabla \rho|}\left[H_{11}+H_{12}+H_{13}\right]\left(\frac{\partial \rho}{\partial x}\right)=\frac{4}{3} \frac{|\nabla \rho|}{\rho^{7 / 3}}\left(\frac{\partial \rho}{\partial x}\right)$,

$\frac{1}{\rho^{4 / 3}|\nabla \rho|}\left[H_{12}+H_{22}+H_{23}\right]\left(\frac{\partial \rho}{\partial y}\right)=\frac{4}{3} \frac{|\nabla \rho|}{\rho^{7 / 3}}\left(\frac{\partial \rho}{\partial y}\right)$,

$\frac{1}{\rho^{4 / 3}|\nabla \rho|}\left[H_{13}+H_{32}+H_{33}\right]\left(\frac{\partial \rho}{\partial z}\right)=\frac{4}{3} \frac{|\nabla \rho|}{\rho^{7 / 3}}\left(\frac{\partial \rho}{\partial z}\right)$.

To connect CPs of $s$ with those of the electron density and with the Laplacian of the electron density we shall consider different cases:

Case 1. AIM-CPs: $\frac{\partial \rho}{\partial x_{i}}=0$ for all $i .|\nabla \rho|$ and $\frac{\partial \rho}{\partial x_{i}}$ are equal to zero along each direction. Thus, there is a division 0/0 for each left hand side of Eqs. 10,11,12. Since $\frac{\partial \rho}{\partial x_{i}}$ approaches to zero faster than $|\nabla \rho|$, this limit is zero along each direction. Therefore, at critical points of $\rho, \nabla s$ tends to zero.

Case 2. Non-AIM-CPs: $|\nabla \rho| \neq 0$,

$$
\begin{aligned}
& {\left[H_{11}+H_{12}+H_{13}\right]\left(\frac{\partial \rho}{\partial x}\right)=\frac{4}{3} \frac{|\nabla \rho|^{2}}{\rho}\left(\frac{\partial \rho}{\partial x}\right),} \\
& {\left[H_{21}+H_{22}+H_{23}\right]\left(\frac{\partial \rho}{\partial y}\right)=\frac{4}{3} \frac{|\nabla \rho|^{2}}{\rho}\left(\frac{\partial \rho}{\partial y}\right),} \\
& {\left[H_{31}+H_{32}+H_{33}\right]\left(\frac{\partial \rho}{\partial z}\right)=\frac{4}{3} \frac{|\nabla \rho|^{2}}{\rho}\left(\frac{\partial \rho}{\partial z}\right) .}
\end{aligned}
$$

Case 2.1. $\frac{\partial \rho}{\partial x_{i}} \neq 0$ for all $i$

$$
\begin{aligned}
& H_{11}+H_{12}+H_{13}=\frac{4}{3} \frac{|\nabla \rho|^{2}}{\rho}, \\
& H_{21}+H_{22}+H_{23}=\frac{4}{3} \frac{|\nabla \rho|^{2}}{\rho}, \\
& H_{31}+H_{32}+H_{33}=\frac{4}{3} \frac{|\nabla \rho|^{2}}{\rho} .
\end{aligned}
$$

Case 2.2. Mutual cancellation of all off-diagonal terms: $H_{12}+H_{13}=H_{21}+H_{23}=H_{31}+H_{32}=0$ $H_{11}=H_{22}=H_{33}=\frac{4}{3} \frac{|\nabla \rho|^{2}}{\rho}$.

It is worth noting that the trace of $H, H_{11}+$ $H_{22}+H_{33}$, which is equal to the Laplacian of the electron density, $\nabla^{2} \rho$, takes positive values and equal to $4 \frac{|\nabla \rho|^{2}}{\rho}$.

Case 2.3. $H$ is diagonal. Then all off-diagonal terms are zero and Equality 19 is obtained.

\section{References}

1. J.P. Perdew, K. Burke, M. Ernzerhof, Physical review letters $77(18), 3865$ (1996)

2. J.M. del Campo, J.L. Gázquez, R.J. AlvarezMendez, A. Vela, International Journal of Quantum Chemistry 112(22), 3594 (2012)

3. A. Zupan, J.P. Perdew, K. Burke, M. Causa, International journal of quantum chemistry 61(5), 835 (1997)

4. H. Schmider, R.P. Sagar, V.H. Smith Jr, Canadian Journal of Chemistry 70(2), 506 (1992)

5. M. Kohout, A. Savin, H. Preuss, The Journal of chemical physics 95(3), 1928 (1991)

6. K. Finzel, Y. Grin, M. Kohout, Theoretical Chemistry Accounts 131(2), 1 (2012)

7. M. Kohout, K. Pernal, F.R. Wagner, Y. Grin, Theoretical Chemistry Accounts: Theory, Computation, and Modeling (Theoretica Chimica Acta) 112(5), 453 (2004)

8. M. Kohout, K. Pernal, F. Wagner, Y. Grin, Theoretical Chemistry Accounts: Theory, Computation, and Modeling (Theoretica Chimica Acta) 113(5), 287 (2005)

9. M. Kohout, F. Wagner, Y. Grin, Theoretical Chemistry Accounts: Theory, Computation, and Modeling (Theoretica Chimica Acta) 119(5), 413 (2008)

10. E.R. Johnson, S. Keinan, P. Mori-Sanchez, J. Contreras-Garcia, A.J. Cohen, W. Yang, Journal of the American Chemical Society 132(18), 6498 (2010)

11. H.J. Bohórquez, R.J. Boyd, Theoretical Chemistry Accounts 127(4), 393 (2010)

12. H.J. Bohórquez, C.F. Matta, R.J. Boyd, International Journal of Quantum Chemistry 110(13), 2418 (2010)

13. P. De Silva, C. Corminboeuf, Journal of chemical theory and computation 10(9), 3745 (2014) 
14. R.A. Boto, J. Contreras-García, J. Tierny, J.P. Piquemal, Molecular Physics pp. 1-9 (2015)

15. A. Cedillo, J. Robles, J.L. Gázquez, Physical Review A 38(4), 1697 (1988)

16. Y. Tal, R. Bader, International Journal of Quantum Chemistry 14(S12), 153 (1978)

17. R.F. Bader, Atoms in molecules (Wiley Online Library, 1990)

18. A.M. Pendás, E. Francisco, M.A. Blanco, C. Gatti, Chemistry-A European Journal 13(33), 9362 (2007)

19. G. Hunter, International Journal of Quantum Chemistry 29(2), 197 (1986)

20. M. Kohout, Molecular Physics 114(7-8), 1297 (2016)

21. M.J. Frisch, G.W. Trucks, H.B. Schlegel, G.E. Scuseria, M.A. Robb, J.R. Cheeseman, G. Scalmani, V. Barone, B. Mennucci, G.A. Petersson, H. Nakatsuji, M. Caricato, X. Li, H.P. Hratchian, A.F. Izmaylov, J. Bloino, G. Zheng, J.L. Sonnenberg, M. Hada, M. Ehara, K. Toyota, R. Fukuda, J. Hasegawa, M. Ishida, T. Nakajima, Y. Honda, O. Kitao, H. Nakai, T. Vreven, J.A. Montgomery, Jr., J.E. Peralta, F. Ogliaro, M. Bearpark, J.J. Heyd, E. Brothers, K.N. Kudin, V.N. Staroverov, R. Kobayashi, J. Normand, K. Raghavachari, A. Rendell, J.C. Burant, S.S. Iyengar, J. Tomasi, M. Cossi, N. Rega, J.M. Millam, M. Klene, J.E. Knox, J.B. Cross, V. Bakken, C. Adamo, J. Jaramillo, R. Gomperts, R.E. Stratmann, O. Yazyev, A.J. Austin, R. Cammi, C. Pomelli, J.W. Ochterski, R.L. Martin, K. Morokuma, V.G. Zakrzewski, G.A. Voth, P. Salvador, J.J. Dannenberg, S. Dapprich, A.D. Daniels, . Farkas, J.B. Foresman, J.V. Ortiz, J. Cioslowski, D.J. Fox. Gaussian09 Revision E.01. Gaussian Inc. Wallingford CT 2009

22. M.W. Schmidt, K.K. Baldridge, J.A. Boatz, S.T. Elbert, M.S. Gordon, J.H. Jensen, S. Koseki, N. Matsunaga, K.A. Nguyen, S. Su, et al., Journal of computational chemistry 14(11), 1347 (1993)

23. J. Ahrens, B. Geveci, C. Law, C. Hansen, C. Johnson. 36-paraview: An end-user tool for large-data visualization (2005)

24. W. Humphrey, A. Dalke, K. Schulten, Journal of molecular graphics 14(1), 33 (1996)

25. A. Fischer, D. Tiana, W. Scherer, K. Batke, G. Eickerling, H. Svendsen, N. Bindzus, B.B. Iversen, The Journal of Physical Chemistry A 115(45), 13061 (2011)

26. A.D. Becke, K.E. Edgecombe, The Journal of chemical physics 92, 5397 (1990)
27. P. Reinhardt, P.E. Hoggan, International Journal of Quantum Chemistry 109(14), 3191 (2009)

28. J. Contreras-García, M. Calatayud, J.P. Piquemal, J. Recio, Computational and Theoretical Chemistry 998, 193 (2012)

29. R. Llusar, A. Beltrán, J. Andrés, S. Noury, B. Silvi, Journal of computational chemistry 20(14), 1517 (1999)

30. S. Shaik, D. Danovich, B. Silvi, D.L. Lauvergnat, P.C. Hiberty, Chemistry-A European Journal 11(21), 6358 (2005)

31. S. Shaik, P. Maitre, G. Sini, P.C. Hiberty, Journal of the American Chemical Society 114(20), 7861 (1992)

32. W. Wu, J. Gu, J. Song, S. Shaik, P.C. Hiberty, Angewandte Chemie 121(8), 1435 (2009)

33. R. Gershoni-Poranne, P. Chen, Chemistry-A European Journal 23(19), 4659 (2017)

34. K. Wagner, M. Kohout, Theoretical Chemistry Accounts 128(1), 39 (2011)

35. J. Contreras-Garcia, W. Yang, in Chemical concepts from density functional theory (Acta PhysicoChimica Sinica.), chap. The chemical information in Jacob's ladder. (To be submitted)

36. N. Gillet, R. Chaudret, J. Contreras-García, W. Yang, B. Silvi, J.P. Piquemal, Journal of chemical theory and computation 8(11), 3993 (2012)

37. D. Fang, R. Chaudret, J.P. Piquemal, G.A. Cisneros, Journal of Chemical Theory and Computation 9(5), 2156 (2013)

38. J. Andrés, S. Berski, J. Contreras-García, P. González-Navarrete, The Journal of Physical Chemistry A 118(9), 1663 (2014)

39. A. Armstrong, R.A. Boto, P. Dingwall, J. Contreras-Garcia, M.J. Harvey, N.J. Mason, H.S. Rzepa, Chemical Science 5(5), 2057 (2014) 